\title{
Qualidade de vida e controle glicêmico do paciente portador de Diabetes Mellitus tipo 2
}

\author{
Quality of life and glycemic control of patients of type 2 Diabetes Mellitus \\ Calidad de vida y el control glucémico de los pacientes de Diabetes Mellitus tipo 2 \\ Alberto José de Amorim Franco Júnior ${ }^{l}$ \\ Maria Geralda Viana Heleno ${ }^{2}$ \\ Universidade Metodista de São Paulo \\ Andressa Pereira Lopes ${ }^{3}$ \\ Universidade Católica de Pernambuco
}

\begin{abstract}
Resumo
Diabetes Mellitus é uma doença crônica degenerativa que impõe uma série de limitações aos seus portadores. Por isso, considerou-se que o estudo da qualidade de vida das pessoas com essa doença pode trazer conhecimento para melhorar as intervenções junto a esses pacientes. O objetivo desta pesquisa foi avaliar a qualidade de vida e o controle glicêmico de portadores de Diabetes Mellitus tipo2. Tratou-se de um estudo descritivo e transversal, desenvolvido com 120 pacientes atendidos no ambulatório de endocrinologia de um hospital situado na cidade de São Bernardo do Campo. Para a coleta dos dados, foram utilizados os seguintes instrumentos: questionário para caracterização da população e questionário de WHOQOL-Bref. Os resultados indicaram que o domínio das relações sociais foi o que mais contribuiu para maior qualidade de vida dos pacientes. Conclui-se que as avaliações médicas devem ser atreladas a avaliações da qualidade de vida para redimensionar as intervenções nestes pacientes.

Palavras-chave: Qualidade de vida; Diabetes mellitus tipo2; Avaliação; Intervenção.
\end{abstract}

\begin{abstract}
Diabetes Mellitus is a chronic degenerative disease that imposes a number of limitations. Therefore, it was considered that the study of the quality of life could bring knowledge to improve interventions for these patients. The objective of this study was to assess quality of life and glycemic control in patients with type 2 Diabetes Mellitus. This was a descriptive, cross-sectional developed with 120 patients attending the outpatient endocrinology clinic of a hospital located in São Bernardo do Campo. To collect data, we used the following instruments: questionnaire to characterize the population, questionnaire WHOQOL-Bref. The results indicated that the domain of social relations was the largest contributor to higher quality of life for patients. We conclude that medical evaluations should be tied to evaluations of the quality of life for resizing interventions with these patients.

Key-words: Quality of life; Diabetes mellitus type 2; Assessment; Intervention.
\end{abstract}

\begin{abstract}
Resumen
La diabetes mellitus es una enfermedad degenerativa crónica que impone una serie de limitaciones. Por lo tanto, se consideró que el estudio de la calidad de vida puede llevar el conocimiento para mejorar las intervenciones para estos pacientes. El objetivo de este estudio fue evaluar la calidad de vida y el control glucémico en pacientes con diabetes mellitus tipo 2. Este fue un estudio descriptivo, de corte transversal desarrollado con 120 pacientes que acudieron a la consulta externa de endocrinología de un hospital ubicado en São Bernardo do Campo. Para recopilar los datos, se utilizaron los siguientes instrumentos: cuestionario para caracterizar la población, el cuestionario WHOQOL-Bref. Los resultados indicaron que el dominio de las relaciones sociales es el que más contribuye a una mayor calidad de vida para los pacientes. Llegamos a la conclusión de que las evaluaciones médicas deben estar vinculados a las evaluaciones de la calidad de vida de las intervenciones de cambio de tamaño con estos pacientes. Palabras-clave: Calidad de vida; Diabetes mellitus tipo 2; Evaluación; Intervención.
\end{abstract}

O Diabetes Mellitus (DM) é uma síndrome de origem múltipla que decorre da falta de insulina ou por efeitos decorridos pela ação ineficaz da insulina. Apresenta como características: hiperglicemia crônica, geralmente acompanhada por dislipidemia;

Endereço 1: 201 SUL, NS 01, CONJUNTO 02 LOTE 01, Plano Diretor Sul. CEP 77015-202 - Palmas- TO

Endereço 2: Rua Dom Jaime de Barros Câmara, 1000, Planalto, CEP: 09895-400 - São Bernardo do Campo - SP

Endereço 3: R. dos coqueiros, 103. Gruta de Lourdes. CEP: 57052-556 - Maceió - AL hipertensão arterial; e disfunção endotelial (American Diabetes Association, 2011, Brasil, 2006). Diabetes Mellitus representa agravo de saúde pública em decorrência do seu caráter crônico (Brasil, 2001).

O DM está entre os quatro mais importantes problemas de saúde (doenças cardiovasculares, neoplasias malignas e causas externas), levandose em consideração o número de pessoas afetadas, a morbidade associada a sua presença, custos envolvidos e redução nos anos de vida (Franco, Mameri, Pagliaro, Iochida \& Goldenberg, 1998). 
Estima-se que, no Brasil, poderão existir cerca de 11 milhões de portadores de DM em 2025 (Brasil, 2006).

Tendo em vista a gravidade desta disfunção, e por se tratar de um problema de saúde pública, foi considerado que o estudo da qualidade de vida pode trazer conhecimentos para melhorar a qualidade das intervenções junto a estes pacientes, levando em consideração, também, a equipe na intervenção, pois essa pode favorecer a melhoria da qualidade de vida do paciente (Heleno, 2001). Na literatura, observase interesse sobre o estudo da qualidade de vida no paciente com diabetes (Beltrame, 2008, Miranzi et al., 2008, Silva et al., 2003, Pais-Ribeiro, 2009, Eren, Erdi \& Sahin, 2008, Abolfotouh, 1999, Aguiar et al., 2008, Mata et al., 2003).

Uma das maneiras de medir a eficácia do controle adequado do diabetes é por avaliação da qualidade de vida. A Organização Mundial de Saúde, a partir do The Whoqol Group (1995, p. 1405), definiu Qualidade de Vida (QV) como "a percepção do indivíduo sobre a sua posição na vida, no contexto da cultura e dos sistemas de valores nos quais ele vive, e em relação a seus objetivos, expectativas, padrões e preocupações". Segundo Silva (2003), sua avaliação se caracteriza como uma relevante maneira de se buscar a melhora nos serviços ligados à saúde, para que possam adequar suas práticas e manter a vida do paciente diabético com qualidade.

O grupo de QV da Organização Mundial de Saúde desenvolveu um instrumento para avaliação da QV, a partir de um projeto que contou com a colaboração de 15 centros simultaneamente em diferentes culturas. $\mathrm{O}$ instrumento resultante foi denominado de WHOQOL-100 (The Whoqol Group, 1998, Chachamovick \& Fleck, 2008). Posteriormente, foi desenvolvido o WHOQOL-bref, que é o instrumento abreviado que consta de vinte e seis questões (Fleck, Xavier, Chachamovich, Vieira, Santos \& Pinzon, 2000).

Em suas diretrizes mais recentes, a Sociedade Brasileira de Diabetes (SBD) adotou a meta de A1C $<6,5 \%$ como indicador de controle glicêmico do diabetes tipo2. Além disso, recomenda-se que os níveis de A1C sejam mantidos nos valores mais baixos possíveis, sem aumentar desnecessariamente o risco de hipoglicemias, particularmente em pacientes insulinizados (Sociedade Brasileira de Diabetes, 2007, 2008, The Diabetes Control And Complications Trial Research Group, 1993).

Em decorrência do exposto, este estudo teve como objetivo avaliar a qualidade de vida e o controle glicêmico em pacientes portadores de diabetes mellitus tipo2. Além disso, espera-se contribuir com reflexões sobre a importância de se levar em consideração os aspectos psicossociais no tratamento do paciente com DM.

\section{Método}

\section{Participantes}

Participou do estudo uma amostra 120 pacientes de ambos os sexos, portadores de diabetes mellitus tipo2, atendidos no ambulatório de endocrinologia de um hospital particular de São Bernardo do Campo.

\section{Instrumentos}

Para coleta de dados, utilizou-se os seguintes instrumentos: questionário sócio demográfico e de dados relacionados ao diabetes; e questionário de qualidade de vida, versão brasileira do WHOQOLBREF (Fleck et al., 2000).

O WHOQOL-BREF apresenta características psicométricas satisfatórias. Em sua versão validada para o Brasil, contou com uma amostra de trezentos indivíduos e mostrou características satisfatórias de consistência interna, validade discriminante, validade de critério, validade concorrente e fidedignidade testereteste. É um instrumento com vinte e seis questões $(\alpha=0,91)$, sendo duas questões gerais de qualidade de vida e as demais vinte e quatro representam cada uma das vinte e quatro facetas do original divididas em quatro domínios: físico, psicológico, relações sociais e meio ambiente.

Os dados sobre a hemoglobina glicosilada foram coletados nos bancos de dados da instituição e posteriormente foram classificados em grupos de "bom controle glicêmico" e "mau controle glicêmico", considerando parâmetros biomédicos Hba1c $\leq 6,8 \%$, conforme recomendações do laboratório que realiza as medidas glicêmicas dos pacientes do Hospital onde foi realizada a pesquisa.

\section{Procedimentos}

O projeto de pesquisa foi avaliado e aprovado pelo comitê de ética em pesquisa da Universidade Metodista de São Paulo, folha de rosto SISNEP (CAAE - 0122.0.214.000-09).

Os participantes foram comunicados sobre a pesquisa após consulta no ambulatório. Em seguida, eram informados sobre os objetivos da pesquisa e depois era lido o Termo de Consentimento Livre e Esclarecido, para que o paciente pudesse ter conhecimento de como seria o processo da pesquisa e, caso aceitasse participar, esse assinava o mesmo termo.

\section{Análise dos dados}

Os dados foram analisados pelo programa estatístico SPSS (Statistical Package for the Social Sciences), versão 17.0, utilizando a estatística descritiva e as provas estatísticas do Qui-Quadrado, Exato de Fisher, Anova, Pos-hoc. O nível de significância adotado foi de 0,05 .

\section{Resultados}


Tabela 1

Descrição do perfil sociodemográfico.

\begin{tabular}{|c|c|c|c|}
\hline \multicolumn{2}{|c|}{ Variáveis } & Frequência & Porcentagem \\
\hline Gênero & $\begin{array}{l}\text { Feminino } \\
\text { Masculino }\end{array}$ & $\begin{array}{l}71 \\
49\end{array}$ & $\begin{array}{l}59,2 \% \\
40,8 \%\end{array}$ \\
\hline Estado Civil & $\begin{array}{l}\text { Casado } \\
\text { Viúvo } \\
\text { Separado } \\
\text { Solteiro }\end{array}$ & $\begin{array}{l}82 \\
29 \\
05 \\
04\end{array}$ & $\begin{array}{c}68,3 \% \\
5,8 \% \\
4,2 \% \\
3,3 \%\end{array}$ \\
\hline Dependentes & $\begin{array}{l}\text { Nenhum } \\
\text { Um } \\
\text { Dois } \\
\text { Três ou mais }\end{array}$ & $\begin{array}{l}55 \\
33 \\
16 \\
16\end{array}$ & $\begin{array}{l}45,8 \% \\
27,5 \% \\
13,3 \% \\
13,3 \%\end{array}$ \\
\hline Escolaridade & $\begin{array}{l}\text { Ensino Fundamental } \\
\text { Ensino Médio } \\
\text { Ensino Superior }\end{array}$ & $\begin{array}{l}90 \\
24 \\
06\end{array}$ & $\begin{array}{c}75 \% \\
20 \% \\
5 \%\end{array}$ \\
\hline
\end{tabular}

\section{Perfil sociodemográfico}

Apartirdaanálisedoquestionáriosociodemográfico foi possível traçar um perfil da amostra dos pacientes atendidos no ambulatório de endocrinologia: maioria formada por mulheres $(59,2 \%)$, estado civil casado $(68,3 \%)$, não dependentes $(45,8 \%)$ e com grau de escolaridade fundamental (75\%). (Tabela 1).

Tabela 1. Inserir aqui: Descrição do perfil sociodemográfico.

\section{Dados relacionados ao diabetes mellitus tipo2}

Quanto aos dados relacionados ao diabetes, os principais resultados apontaram que: em sua maioria, os pacientes possuíam o diagnóstico de diabetes há mais de dez anos (43,3\%), faziam uso regularmente de medicação $(95,8 \%)$, não utilizava insulina $(80 \%)$, faziam dieta $(85,8 \%)$ e apresentavam bom controle glicêmico (53,3\%) (Tabela 2).

Tabela 2. Inserir aqui: Dados relacionados ao diabetes.

\section{Avaliação da qualidade de vida}

Os escores obtidos por meio de 120 participantes para os domínios de qualidade de vida foram: domínio físico - valor mínimo $(98,57)$, máximo $(17,71)$, média $(13,042)$ e desvio padrão $(1,703)$. Domínio psicológico - valor mínimo $(8,00)$, máximo $(17,33)$, média $(13,383)$, desvio padrão $(1,927)$. Domínio relações sociais - valor mínimo $(4,00)$, máximo $(20,0)$, média $(14,322)$, desvio padrão $(2,991)$. Domínio meio ambiente - valor mínimo $(8,00)$, máximo $(18,50)$, média $(13,225)$ e desvio padrão $(2,054)$ (Tabela 3$)$.

Tabela 3. Inserir aqui: Descrição dos domínios de Qualidade de Vida.

Analisando a associação entre as características sociodemográficas e qualidade de vida identificou-se que:

Existe uma diferença significativa (ANOVA e
Pos hoc) quando comparados com o estado civil com a qualidade de vida dentro do domínio físico (média=15,4286 $(\mathrm{DP} \pm=1,937)$ e $\mathrm{p}=0,001)$. Estes resultados evidenciaram que os participantes que são separados apresentam melhor qualidade de vida no domínio físico.

Quanto ao número de dependentes, os resultados mostraram a existência de uma diferença significativa (ANOVA e Pos hoc) no domínio das relações sociais (média $=12,250 \quad(\mathrm{DP} \pm=3,991)$ e $\mathrm{p}=0,025)$ de qualidade de vida. Estes resultados mostraram que aqueles com três ou mais dependentes, têm melhor qualidade de vida no domínio das relações sociais.

Sobre a escolaridade, os resultados apontaram uma diferença significativa (ANOVA e Pos hoc) no domínio psicológico (média $=14,194(\mathrm{DP} \pm=1,437)$ e $p=0,009)$ da qualidade de vida. Estes resultados mostraram que os participantes com ensino médio possuem melhor qualidade de vida no domínio psicológico.

Analisando a associação entre os dados relacionados ao diabetes e qualidade de vida identificou-se que:

Quanto à insulinoterapia, os resultados apontaram uma diferença significativa (ANOVA e Pos hoc) no domínio psicológico (média $=13,583(\mathrm{DP} \pm=1,833)$ e $\mathrm{p}=0,022$ ) e domínio relações sociais (média $=14,680$ $(\mathrm{DP} \pm=2,677)$ e $\mathrm{p}=0,008)$ da qualidade de vida. Estes resultados mostraram que os participantes que não usam insulina apresentam melhor qualidade de vida no domínio psicológico e relações sociais.

Por intermédio do teste Qui-quadrado, constatouse uma diferença significante entre tempo de diagnóstico e controle glicêmico $(\mathrm{p}=0,002)$. Isso significa que, quanto maior o tempo de diagnóstico, pior o controle glicêmico.

Por intermédio do teste exato de Fisher, contatouse que existe diferença significante entre seguir dieta 
Tabela 2

Dados relacionados ao diabetes.

\begin{tabular}{lcc}
\hline \multicolumn{1}{c}{ Variáveis } & Frequência & Porcentagem \\
\hline Tempo de Diagnóstico & 52 & $43,3 \%$ \\
Mais de 10 anos & 25 & $20,8 \%$ \\
1 a 2 anos & 19 & $15,8 \%$ \\
3 a 5 anos & 12 & $10,0 \%$ \\
Até 11 meses & 12 & $10,0 \%$ \\
6 a 9 anos & & \\
Medicação & 115 & $95,8 \%$ \\
Regularmente & 05 & $4,2 \%$ \\
Não & & \\
Insulinoterapia & 96 & $80,0 \%$ \\
Não & 24 & $20,0 \%$ \\
Sim & & \\
Dieta & 103 & $85,8 \%$ \\
Sim & 17 & $14,2 \%$ \\
Não & & \\
Controle Glicêmico & 64 & $53,3 \%$ \\
Bom & 56 & $46,7 \%$ \\
Mau & & \\
\hline
\end{tabular}

$(p=0,008)$ e controle glicêmico. Isso significa que os participantes que seguem dieta apresentam bom controle glicêmico.

Houve associação no teste exato de Fisher entre fazer uso de medicação $(p=0,040)$ e controle glicêmico. Ou seja: aqueles que fazem uso de medicação regularmente também apresentam bom controle glicêmico. Por fim, pacientes que têm diabetes há dez anos ou mais tendem a ter pior controle glicêmico.

\section{Discussão}

Os participantes deste estudo foram pacientes com Diabetes Mellitus tipo2 atendidos por um convênio médico com rede própria, portanto, pacientes com atendimento médico ambulatorial regular. Era esperado que estes pacientes estivessem com um controle adequado da glicemia, considerando os recursos oferecidos pelo atendimento médico, mas observou-se que $46,7 \%$ deles não conseguem manter um bom controle glicêmico. Este fato determinará a piora de seus estado de saúde, com o aumento das taxas de doenças associadas (American Diabetes Association, 2011, Sociedade Brasileira de Diabetes, 2007, The Diabetes Control And Complications Trial Research Group, 1993).

$\mathrm{Na}$ avaliação dos domínios de qualidade de vida, os participantes obtiveram a melhor média $(14,322)$ no domínio das relações sociais. Este domínio é composto por relações pessoais, suporte (apoio) social e vida sexual. Pode-se afirmar que estes pacientes possuem boas relações pessoais e sentem que são apoiados socialmente. Isto é de grande relevância, pois aponta a possibilidade dessas pessoas conseguirem uma boa qualidade de vida no domínio das relações sociais, apesar da doença e do tratamento.

Estes resultados estão de acordo com os obtidos por Beltrame (2008) e Miranzi et al. (2008), em seus estudos sobre qualidade de vida com portadores de Diabetes Mellitus tipo2. Portanto, o domínio das relações sociais foi o que mais contribuiu com a boa qualidade de vida dos pacientes com diabetes. Este fato pode ser associado à importância de uma boa relação com os membros da equipe do hospital, familiares e amigos.

No presente estudo, quando comparados os domínios da qualidade de vida ao estado civil, observou-se que os participantes da amostra que eram separados apresentaram melhor qualidade de vida no domínio físico. Este resultado mostra que, mesmo não contando com um cônjuge que pode ser um fator de ajuda no auto-cuidado, no apoio e na adesão ao tratamento, os mesmos sentem-se satisfeitos quanto aos aspectos do domínio físico: dor e desconforto, energia e fadiga, sono e repouso. As amizades podem exercer influência positiva na qualidade de vida dos pacientes com DM. (Silva et al., 2003). Pode-se levantar a hipótese de que, mesmo sem poder contar com cônjuges que poderiam lhes prover suporte social e contribuir na percepção da qualidade de vida, estes pacientes podem contar com amigos, ou pessoas que fazem parte de suas redes sociais, que lhes permitem perceber satisfação em relação a qualidade de vida. Incluem-se nessa lista os profissionais da saúde que fazem parte da equipe de atendimento ao paciente com diabetes (Heleno, 2001). 
Tabela 3

Descrição dos domínios de Qualidade de Vida.

\begin{tabular}{lccccc}
\hline \multicolumn{1}{c}{$\begin{array}{c}\text { Variáveis } \\
\text { Qualidade de Vida }\end{array}$} & $N$ & Mínimo & Máximo & Média & Desvio Padrão \\
\hline Domínio Físico & 120 & 8,57 & 17,71 & 13,042 & 1,703 \\
Domínio Psicológico & 120 & 8,00 & 17,33 & 13,383 & 1,927 \\
Domínio Relações Sociais & 120 & 4,00 & 20,00 & 14,322 & 2,991 \\
Domínio Meio Ambiente & 120 & 8,00 & 18,50 & 13,225 & 2,054 \\
\hline
\end{tabular}

Foi constatado nos resultados que os participantes que moram com três ou mais dependentes apresentam melhor qualidade de vida no domínio das relações sociais. Estes resultados mostram que possivelmente suas relações familiares contribuem para sua satisfação nas relações pessoais e podem atuar positivamente em relação ao controle glicêmico. A avaliação e compreensão das relações sociais, aspectos clínicos e controle glicêmico possibilitam gerenciar variáveis que atuam positivamente ou negativamente na aquisição da qualidade de vida (Pais-Ribeiro, 2009, Beltrame, 2008).

Outro resultado obtido nesta amostra foi a associação entre escolaridade e domínio psicológico. Assim, quanto melhor o nível de escolaridade, melhor a qualidade de vida, considerando o domínio psicológico. Supõem-se que ter a escolaridade até o ensino médio pode repercutir positivamente na compreensão das informações recebidas pela equipe, elevando a auto-estima e sentimentos positivos diante do tratamento e contribuindo para postergar ou evitar o aparecimento das complicações crônicas. Estes dados estão de acordo com os obtidos por Eren, Erdi \& Sahin, (2008), que apontaram em seus estudos associação entre escolaridade e melhor qualidade de vida em pacientes com Diabetes Mellitus tipo2.

Outro dado obtido foi que a não utilização da insulinoterapia no tratamento realizado nos participantes deste estudo foi associado a uma melhor qualidade de vida (domínio psicológico e relações sociais). Em relação a esse resultado, pode-se supor que, por não necessitarem de insulina em seus tratamentos, estes pacientes avaliam o fato como positivo, tendo repercussões em sua auto-estima, por associarem o uso de insulina ao controle metabólico inadequado. Além disso, esses resultados mostram que os pacientes se sentem melhor socialmente quando não utilizam insulina. Estes dados são corroborados por Aguiar et al. (2008) e Mata et al. (2003), quando mostram que, dentre as variáveis que podem interferir na qualidade de vida, pode-se destacar o uso da insulina, relacionado aos aspectos psicológicos que devem ser levados em consideração na promoção da qualidade de vida, inclusive sentimentos ruins relativos à dependência (Pais-Ribeiro, 2009).

Alguns fatores são apontados como fundamentais para a manutenção de um bom controle glicêmico
(Brasil, 2001, 2006, Abolfotouh, 1999). Entre eles, foram analisados neste estudo o tempo de duração da doença, atividade física, terapia nutricional e medicação. A análise dos dados mostrou que não existe, nesta amostra, relação entre controle glicêmico e atividade física. Entretanto, foi observado que, de fato, quanto maior o tempo da doença, pior o controle glicêmico.

Ao que se refere à terapia nutricional, em relação ao realizar ou não dieta, os dados deste estudo mostram que os pacientes com bom controle glicêmico fazem algum tipo de dieta. Este fato aponta a possibilidade de controle de peso e redução do risco de complicações crônicas. Esse resultado é semelhante ao encontrado no estudo de Beltrame (2008): a dieta planejada funciona como um fator importante no controle de complicações crônicas (American Diabetes Association, 2011). Cabe lembrar que, dos participantes desta pesquisa, apesar de $46,7 \%$ fazerem dieta, não conseguem manter um bom controle glicêmico. No atendimento composto por uma equipe multiprofissional ao paciente com DM, o tratamento nutricional é fundamental para o controle glicêmico e deve ser implantado levando em consideração a singularidade de cada caso. Para isto, a equipe deve desenvolver adequadamente uma relação positiva com o paciente, de modo que este suporte social contribua para a melhora do paciente e de sua qualidade de vida (Heleno, 2001).

Verificou-se que os participantes desta pesquisa que usam medicação regularmente apresentaram bom controle glicêmico. Este resultado aponta o uso de medicação sob a orientação adequada da equipe e levando em conta que a singularidade do paciente possibilita o bom controle dos níveis de glicemia. Devese atentar que no paciente com diabetes tipo2, caso o nível glicêmico desejado não tenha sido atingido com o emprego de medidas dietéticas, exercícios ou com o uso de medicamentos anti-diabéticos, a combinação de diferentes medicações com diferentes mecanismos de ação é comprovadamente útil no tratamento e deve ser utilizada (Sociedade Brasileira de Diabetes, 2007). Cabe aqui destacar que o uso de medicação adequada e controle glicêmico satisfatório foram observados nesta pesquisa, fato que contribui para retardar o aparecimento de complicações crônicas nestes pacientes. Com isso, o uso de medicação pode 
reduzir pela metade o aparecimento de complicações do diabetes (Brasil, 2006).

\section{Considerações Finais}

Os participantes deste estudo eram pacientes com Diabetes Mellitus tipo2 atendidos por um convênio médico com rede própria e, apesar da qualidade do atendimento médico ambulatorial e regular, aproximadamente $50 \%$ deles não apresentam bom controle glicêmico. Este resultado leva-nos à reflexão sobre o papel da equipe no atendimento ao paciente com diabetes. Embora o atendimento médico seja de muita importância, os dados deste estudo apontam para a necessidade de o atendimento ser realizado por uma equipe multiprofissional. A presença de profissionais da área de nutrição, enfermagem, psicologia, educação e educação física poderia melhorar consideravelmente a qualidade do controle glicêmico e principalmente da qualidade de vida desses pacientes.

Os profissionais da saúde precisam ficar atentos aos fatores médicos e às variáveis psicossociais, pois são fatores que interferem na qualidade de vida e na qualidade do tratamento. Portanto, estas variáveis devem ser controladas no curso do tratamento e poderão contribuir para a eficiência das intervenções e promoção de saúde de pacientes com DM tipo2.

Conhecer as dimensões da qualidade de vida mais afetadas ou que apresentam maiores escores possibilita o planejamento de ações de promoção da saúde e prevenção de complicações. Assim, é recomendado ao ambulatório a implantação de uma equipe multiprofissional, que possa acompanhar estes pacientes, proporcionando um ganho na qualidade do tratamento e, principalmente, na vida destes pacientes, incluindo neste acompanhamento a avaliação da qualidade de vida.

\section{Referências}

Abolfotouh, M. A. (1999). Effect of diabetes mellitus on quality of life: a review. Annals of Saudi Medicine, 19 (6), 518-524.

Aguiar, C. C. T., Vieira, A. P. G. F., Carvalho, A. F. \& Montenegro-Junior, R. M. (2008). Instrumentos de avaliação de qualidade de vida relacionados à saúde no diabetes melito. Arq. Bras. Endocrinol. Metab, 52 (6), 931-939.

American Diabetes Association (2011). Standards of Medical Care in Diabetes. Diabetes Care, 34 (1), 11-61.

Beltrame, V.(2008). Qualidade de vida de idosos diabéticos. 100f. Tese (Doutorado em Gerontologia Biomédica)-Instituto de Geriatria e Gerontologia, Pontifícia Universidade Católica do Rio Grande do Sul.

Brasil (2006). Ministério da Saúde. Secretaria de Atenção à Saúde. Departamento de Atenção Básica. Diabetes Mellitus. Brasília : Ministério da Saúde.

Brasil (2001). Ministério da Saúde. Secretaria de Políticas de Saúde. Plano de reorganização da atenção à hipertensão arterial e ao diabetes mellitus: manual de hipertensão arterial e diabetes mellitus. Brasília: Ministério da Saúde.

Chachamovick, E. \& Fleck, M. P. A. (2008). Desenvolvimento do WHOQOL-BREF. In Fleck, M. P. A. et al.. A avaliação da qualidade de vida, ( $1^{\mathrm{a}}$ ed.). Porto Alegre: Artmed.
Eren, I., Erdi, O. \& Sahin, M. (2008). The effect of depression on quality of life of patients with type II Diabetes Mellitus. Depression and anxiety (25), 98-106.

Fleck, M. P. A. \& Xavier, S. L. M., Chachamovich, E., Vieira, G., Santos, L., Pinzon, V. (2000). Aplicação da versão em português do instrumento abreviado de avaliação de qualidade de vida WHOQOL-BREF. Rev. Saúde Pública, 34 (2),178-183.

Franco, L. J., Mameri, C., Pagliaro, H.; Iochida, L. C., \& Goldenberg, P. (1998). Diabetes como causa básica ou associada de morte no Estado de São Paulo, Brazil, 1992. Rev. Saúde Publica, São Paulo, 32 (3), 237-245.

Heleno, M. G. V. (2001). Organizações patológicas e equilíbrio psíquico em pacientes com diabetes tipo II. Mudanças, 9 (15), 75 158.

Mata, C. M., Roset, G. M., Badia, L. X., Antonanzas, V. F. \& Ragel, A. J. (2003). Effect of type-2 diabetes mellitus on the quality of life of patients treated at primary care consultations in Spain. Aten Primaria, 31 (8), 493-499.

Miranzi, S. S. C., Ferreira, F. S., Iwamoto, H. H., Pereira, G. A. \& Miranzi, M. A. S. (2008). Qualidade de vida de indivíduos com diabetes mellitus e hipertensão acompanhados por uma equipe de Saúde da Família. Texto Contexto Enfermagem, 17 (4), 672-679.

Pais - Ribeiro, J. L. (2009). A importância da qualidade de vida para a psicologia da saúde. In: Cruz, J. P.; Jesus, S. N. Nunes, C. Bem-estar e qualidade de vida. Alcochete: Textiverso.

Silva, M. J. M. (2003). Avaliação da qualidade de vida de portadores de diabetes melito. 2003.101f. Dissertação (Mestrado em Psicologia)-Centro de Filosofia e Ciências Humanas, Universidade Federal de Santa Catarina.

Silva, I., Pais-Ribeiro, J., Cardoso, H., Ramos, H., Carvalhosa, S. F.; Dias, S. \& Gonçalves, A. (2003). Efeitos do apoio social na qualidade de vida, controle metabólico e desenvolvimento de complicações crônicas em indivíduos com diabetes. Psicologia, Saúde \& Doença, 4 (1), 21-32.

Sociedade Brasileira de Diabetes (2009, Outubro 29). Diretrizes da sociedade brasileira de Diabetes, 2008. Acessado de http://www.diabetes.org.br/educacao/docs/Diretrizes_SBD_2008_ MAR 12.pdf.

Sociedade Brasileira de Diabetes (2007). Diretrizes da sociedade brasileira de diabetes: tratamento e acompanhamento do Diabetes Mellitus, (1 $1^{\mathrm{a} e d}$.). Rio de Janeiro: Diagraphic.

The Diabetes Control And Complications Trial Research Group (1993). The effect of intensive treatment of diabetes on the development and progression of long-term complications in insulin-dependent diabetes mellitus. N Engl J Med, 329 (14), 977986.

The Whoqol Group (1998). The world health organization quality of life assessment (WHOQOL): development and general psychometric prosperties. Soc. Sci. Med., 46 (12), 1569-1585.

THE WHOQOL GROUP (1995). The World Health Organization quality of life assessement (WHOQOL): proposition paper from the World Health Organization. Soc. Sci. Med., (41), 1447-1462. 
Sobre os autores:

Alberto José de Amorim Franco Júnior - Mestre em Psicologia da Saúde pela Universidade Metodista de São Paulo - UMESP, Psicólogo do Hospital Geral Público de Palmas- HGPP.

E-mail: albertofraanco@gmail.com

Maria Geralda Viana Heleno - Doutora em Psicologia Clínica pela Universidade de São Paulo - USP, Docente do curso de Mestrado em Psicologia da Saúde da Universidade Metodista de São Paulo - UMESP. E-mail: maria.heleno@metodista.br

Andressa Pereira Lopes - Doutoranda em Psicologia Clínica pela Universidade Católica de Pernambuco (UNICAP), Mestre em Psicologia da Saúde pela Universidade Metodista de São Paulo - UMESP.

E-mail: andressa_lopes@hotmail.com 